\title{
Diffractive/refractive optics for high energy astronomy
}

\section{Gamma-ray phase Fresnel lenses}

\begin{abstract}
G. K. Skinner*
1 School of Physics and Astronomy, University of Birmingham, Edgbaston, Birmingham, B15 2TT, England, UK

2 CESR, 9 avenue du Colonel Roche, 31028 Toulouse, France

Received 30 March 2001 / Accepted 22 May 2001

Abstract. Diffractive optics components such as Fresnel Zone Plates and their derivatives potentially form the basis for telescope systems for X-ray and gamma-ray astronomy with high sensitivity and superb angular resolution. The main problem is that systems with convenient design parameters involve very long focal lengths. The design considerations and performance of telescopes using a simple Phase Fresnel Lens on one spacecraft and a detector assembly on another are considered. Such systems are shown to have the potential to provide orders of magnitude improvement on currently available sensitivity. At the same time the angular resolution can be at the micro arcsecond level or better - sufficient to resolve the structure surrounding the event horizon of black holes in nearby galaxies.
\end{abstract}

Key words. telescopes - methods: observational - techniques: interferometric - gamma-rays: observations X-rays: general

\section{Introduction}

Observations at hard X-ray and gamma-ray energies are severely constrained by the limited sensitivity and poor angular resolution of the present generation of instrumentation. The sensitivity could be enormously improved if gamma-ray radiation could be concentrated from a large collection area onto a small, low background detector, as is done with grazing incidence optics at lower energies in, for example, the Chandra and XMM-Newton observatories. Similarly, if a diffraction-limited gamma-ray optical system, even of modest diameter (a few metres), could be constructed, then the angular resolution achievable would be a fraction of one micro arcsecond $\left(\mu^{\prime \prime}\right)$. This would be better than that achievable in any other waveband and would be sufficient to resolve the structure in the emitting region immediately surrounding the event horizon of massive black holes in the nucleii of nearby galaxies.

The object of this paper is to point out that in principle simple diffractive optics in the form of a Phase Fresnel Lens can simultaneously achieve both of these advances. This idea has been briefly introduced (Skinner 2001b) but is here examined in detail. The background to the problem of obtaining high sensitivity and ultra-high angular resolution observations is first discussed (Sect. 2) and the concept of utilising diffractive optics at gamma-rays is then introduced (Sect. 3), along with some specific considerations for astronomical applications (Sect. 4). The practical

\footnotetext{
* e-mail: skinner@cesr.fr
}

problems are discussed in Sect. 5 and the performance anticipated and the science which it would make possible are reviewed in Sect. 6. In a companion paper (Skinner 2001a, Paper II) some variations of the proposed configuration, which offer advantages in particular circumstances, will be discussed.

\section{Background and context}

At X-ray energies the present generation of X-ray instrumentation for astronomy depends on grazing angle reflection optics in a nested Wolter-I configuration. In this way the Chandra mission offers angular resolution of 0.5 arcsec (Jerius et al. 2000) and, with thinner, less precise, optics XMM-Newton obtains an effective area of more than $0.4 \mathrm{~m}^{2}$ (Stockman et al. 1999). Both of these instruments are limited to photon energies below $\sim 10 \mathrm{keV}$.

$\mathrm{X}$-ray missions actively under study, in particular Constellation-X (White \& Tananbaum 1999) and Xeus (Bavdaz et al. 1999), will make possible considerable improvements in sensitivity and spectral resolution. They will not emphasise angular resolution, which will remain $10^{3}-10^{6}$ times worse than the diffraction limit. At X-ray energies only interferometry, discussed below, offers the prospect of ultra-high angular resolution.

Moving to hard X-rays and gamma-rays, the sensitivity of the current generation of instruments - ComptonGRO and Integral - is very much poorer than that obtained in the X-ray band. This is largely because it is 
not currently possible to concentrate the flux as is done at lower energies with mirror optics. The imaging techniques, too, are indirect and have poor angular resolution - of the order $0.1-10^{\circ}$.

Grazing incidence optics with multilayer coatings are being actively pursued by a number of groups for focussing energies up to about $100 \mathrm{keV}$ (e.g. Yamashita et al. 1998; Ogasaka et al. 2000; Craig et al. 1998; Hussain et al. 1999; Mao et al. 1999). In principle such techniques can focus radiation of even higher energies, though the number of layers for efficient reflection would become very large and the grazing angles very small, so the area of coated surface for a given effective area would be huge. The angular resolution of multilayer mirrors depends on the precision of the surface of the substrate and of the coating. At present it is little better than one arc minute and there seems little prospect either of achieving large effective areas or of approaching the diffraction limit using this technology.

MAXIM is a mission currently under study that would use X-ray interferometry to allow images to be reconstructed with a resolution corresponding to a baseline of $1 \mathrm{~m}$ (pathfinder version) or even $100 \mathrm{~m}$ ("event horizon" version). Using X-rays of $\sim 1$ to $6 \mathrm{keV}$ respectively this results in resolutions of 100 and $0.1 \mu^{\prime \prime}$ (Cash et al. 2000). MAXIM can be regarded as a large modified Wolter III telescope with unfilled aperture in which the sampled parts of the aperture are approximated by individual plane surfaces. To get sub micro arcsecond resolution at keV energies requires an entrance aperture of hundreds of metres and the idea is that the primary mirror segments would be on 32 separate spacecraft and the secondary mirror segments and detector array on two more.

In the gamma-ray band a first step towards attaining flux concentration has recently been made through the use of Laue diffraction in Ge-Si crystals in a telescope for gamma-rays of $170 \mathrm{keV}$ (Laporte et al. 1999) and this technology has been proposed for part of the HXT for the Constellation-X mission (Gorenstein et al. 1996). Concentric rings of carefully oriented crystals diffract the incoming flux towards a common focal point, each ring using a different set of crystal planes. This technique is expected to become important as a way of collecting flux onto a small detector but extremely high angular precision would only be obtained with very carefully aligned, highly perfect, crystals, that have an extremely narrow band-pass. Furthermore the approach does not lend itself to true imaging.

Other approaches to gamma-ray imaging for astronomy and their limitations have been reviewed by Skinner (2001b). None apart from the use of diffraction and/or refraction appear to offer the prospect of extremely high angular resolution and sensitivity. Purely refractive optics could be considered in some circumstances, but Yang (1993) has shown how absorption limits the effective area possible. Systems in which diffraction plays the major role turn out to be preferable and it is on such designs that we will concentrate here.
Diffractive lenses in the form of Fresnel Zone Plates (FZPs) were used in early solar X-ray imaging (Kraemer et al. 1978), but since then have not found application in high energy astronomy, due to their low efficiency and small apertures. Masks with FZP patterns cut into them have been proposed for astronomy both as simple coded masks (Mertz \& Young 1961) and in pairs in a modulation collimator configuration in which Moiré fringes are recorded (Desai et al. 1998). Such systems, however, have no flux concentrating capability and are quite distinct from those advocated here. What is discussed here is the possible application to gamma-ray astronomical applications of true diffractive lenses with large effective area.

\section{Gamma-ray diffractive optics}

MAXIM will offer major advances in the X-ray band and is technically so challenging that it may seem premature to consider how comparable resolution might be obtained at still higher energies, where performance is currently far inferior to that possible at lower energies. But this paper aims to show that this may be feasible and that, although there are some disadvantages of working at higher energies, in other respects things actually become simpler.

Fresnel Zone Plates (FZPs, Fig. 1a), as invented by Soret (1875), are in some respects related to the Laue lens mentioned in Sect. 2 as each small region of an FZP can be thought of as a small grating that diffracts the incoming radiation towards the focus, the pitch of the diffraction grating becoming smaller as higher deflection angles are needed at larger radii.

A simple FZP has a maximum efficiency of $1 / \pi^{2}=$ $10.1 \%$. This results from the fact that apart from the first order $(n=1)$ focus, energy also passes unfocussed $(n=0)$, to higher orders $(n=3,5 \ldots)$ and to virtual focii $(n=-1,-3,-5 \ldots)$.

As well as independently proposing the FZP, Rayleigh pointed out the possibility, demonstrated by Wood (1898), of obviating the zero order loss in what can be termed the Phase Zone Plate (PZP, Fig. 1b). The opaque regions are replaced by ones that allow the radiation to pass, but which have a thickness of refractive material such as to impose a phase shift of $\pi$. The theoretical efficiency limit is raised to $4 / \pi^{2}=40.4 \%$.

Ideally the thickness, and hence the phase change, within each zone should be a continuous function of radius. This leads to the kineform, or Phase Fresnel Lens (PFL) (Miyamoto 1959) (Fig. 1c), in which ideally all of the power is directed into order $n=1$, giving an efficiency of up to $100 \%$. The same effect can be achieved with a graded refractive index instead of varying thickness (Fujisaki \& Nakagiri 1990; Yang 1993). Stepped approximations to a PFL (di Fabrizio \& Gentili 1999) can still have efficiencies approaching this value. Effectively the graded diffraction grating is blazed using refraction to concentrate all of the power into order $n=1$.

FZPs, PZPs and PFLs are increasing being used for X-ray microscopy and other applications at electron 


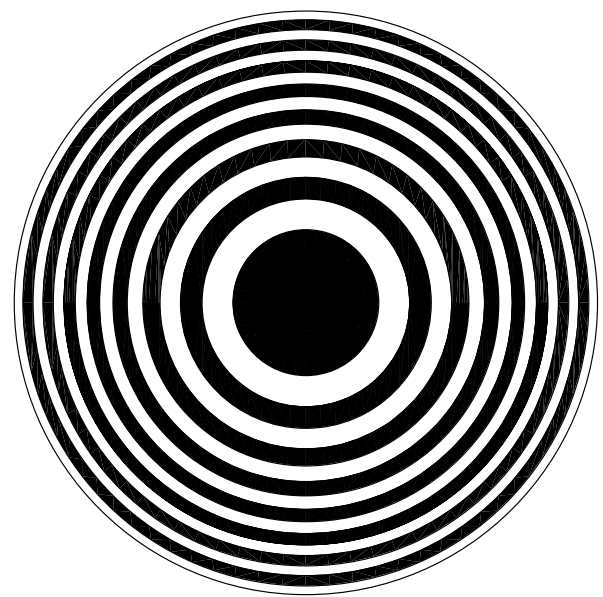

(a)

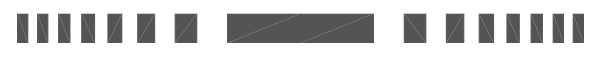

(b)

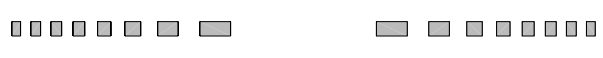

(c)

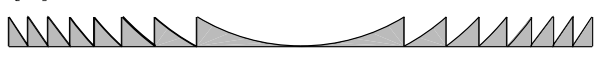

Fig. 1. a) A Fresnel Zone Plate (FZP), b) A Phase Zone Plate $(\mathrm{PZP}), \mathbf{c})$ A Phase Fresnel Lens (PFL).

synchrotron facilities. Some example parameters for lenses that are based on these principles and that have been used for laboratory applications are given in Table 1.

In view of the need for high efficiency, we will concentrate here on PFLs, and hence the refractive index of the material from which the lens is made is a critical parameter. The refractive index of a material at X-ray/gammaray energies is usually expressed as

$n *=(1-\delta)-i \beta$.

The real part of $n *$ is slightly less than unity, corresponding to values of $\delta$ which are small and positive. $\delta$ can be expressed in terms of the real component $f_{1}$ of the form factor,

$\delta=\frac{r_{\mathrm{e}}}{2 \pi} n_{\mathrm{a}}\left(\frac{h c}{E}\right)^{2} f_{1}$,

where $r_{\mathrm{e}}$ is the classical electron radius, $n_{\mathrm{a}}$ the number density of atoms, and $E$ the energy. Similarly the imaginary component of $n *$, which corresponds to absorption, can be written

$\beta=\frac{r_{\mathrm{e}}}{2 \pi} n_{\mathrm{a}}\left(\frac{h c}{E}\right)^{2} f_{2}, \quad f_{2}=E \frac{\mu_{E}}{2 h c r_{\mathrm{e}}}$,

where $\mu_{E}$ is the absorption cross-section and $E$ the photon energy.

Well above absorption edges, and apart from small relativistic and nuclear corrections, $f_{1}$ is essentially constant

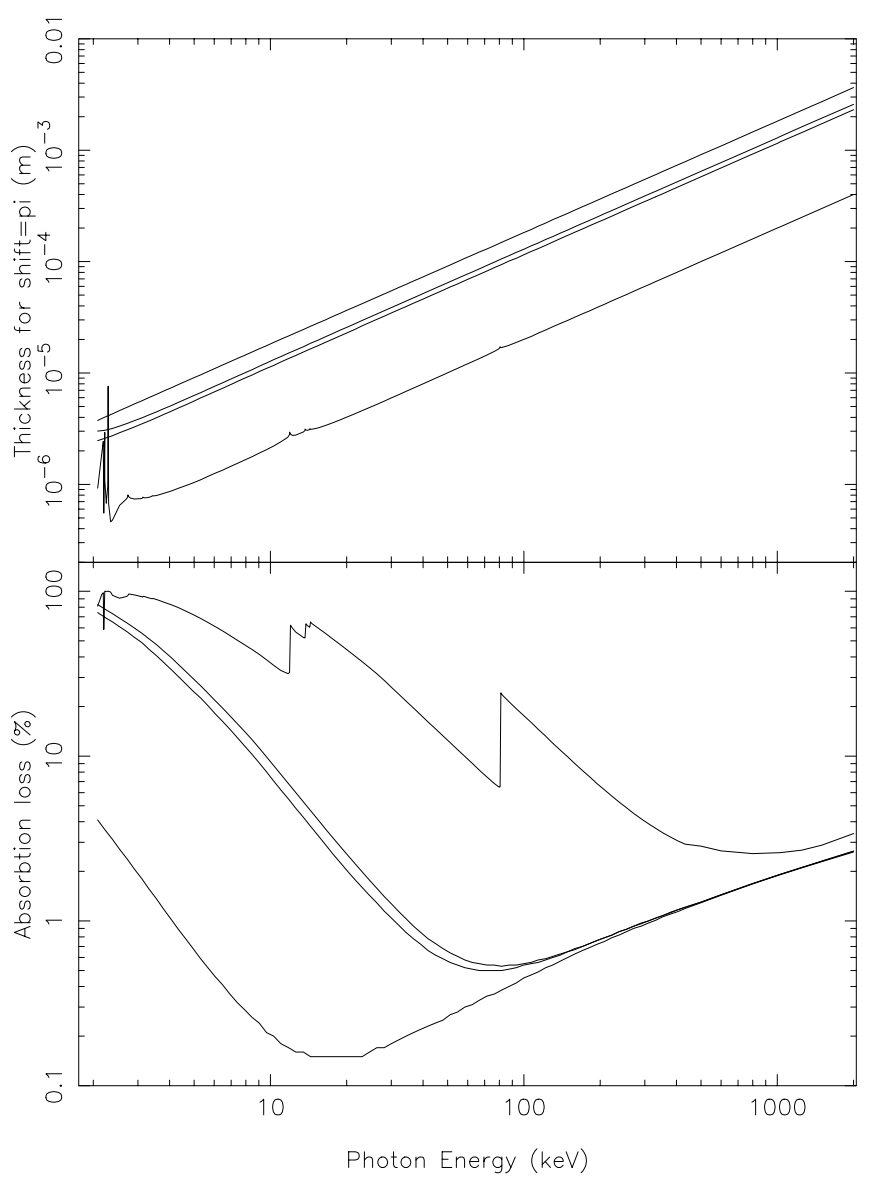

Fig. 2. The thickness, $t_{\pi}$, of example materials necessary to produce a phase shift of $\pi$, and the corresponding absorption losses. The materials represented are $\mathrm{Be}, \mathrm{Si}, \mathrm{Ni}$ and $\mathrm{Au}$ (from top to bottom and from bottom to top respectively in the two plots). Based on data from Chantler (1995).

and equal to the number of electrons per atom, $Z$. It follows that the thickness $t_{\pi}$ necessary to provide a phase shift of $\pi$ is approximately proportional to energy. The thickness of a PZP or the mean thickness of a PFL will be equal to $t_{\pi}$. Values of $t_{\pi}$ for some example materials are shown in Fig. 2. Much of the present work depends on the fact that as one moves up in energy from the X-ray band, although $t_{\pi}$ increases, the associated absorption becomes smaller and over a wide range of energy the losses fall to a low level (Fig. 2).

The focal length, $f$, the finest pitch of the pattern, $p$, and the lens diameter, $d$, are inter-related; we review first some of the considerations which dictate the choice of these parameters.

For microscopy, very fine pitch patterns are sought because the diffraction limited spatial resolution in the object plane is $s_{\mathrm{d}}=1.22 \lambda \mathrm{f} / \mathrm{d}=1.22 \lambda F_{\text {no }}=0.61 p$. Here the resolution is that given by the Rayleigh critereon, which corresponds to the $60 \%$ power diameter.

For astronomy, the diffraction limited image spot size is equal to $s_{\mathrm{d}}$, as given by the above expressions, but it is the angular resolution $\theta_{\mathrm{d}}=s_{\mathrm{d}} / f$ that is important. From the points of view of the angular resolution of a 
Table 1. Properties of some diffractive lenses used in laboratory systems and of some example systems of the type proposed here for gamma-ray astronomy.

\begin{tabular}{|c|c|c|c|c|c|c|}
\hline \multirow{2}{*}{$\frac{\text { Reference }}{\text { Energy }(\mathrm{keV})}$} & $\begin{array}{l}\text { Example } \\
\text { (a) }\end{array}$ & $\begin{array}{l}\text { eported labor } \\
\text { (b) }\end{array}$ & $\begin{array}{l}\text { atory systems } \\
\text { (c) }\end{array}$ & \multicolumn{3}{|c|}{$\begin{array}{c}\text { Example diffractive lens } \\
\text { telescopes }\end{array}$} \\
\hline & 0.4 & 8,20 & 8 & 200 & 500 & 847 \\
\hline Type & FZP & $\mathrm{PZP}$ & Stepped PFL & \multicolumn{3}{|c|}{ PFL } \\
\hline Material & $\mathrm{Ge}$ & $\mathrm{Au}$ & $\mathrm{Ni}$ & \multicolumn{3}{|c|}{$\mathrm{Al}$} \\
\hline Maximum thickness, $t(\mu \mathrm{m})$ & 0.18 & $1.6,3$ & 4.5 & 450 & 1200 & 1900 \\
\hline Shortest period, $p(\mu \mathrm{m})$ & 0.06 & 0.5 & 2 & 2500 & 1000 & 590 \\
\hline Aspect ratio $(t / p)$ & 3 & $3.2,6$ & 2.25 & 0.5 & 1.2 & 2.0 \\
\hline Focal length $f(\mathrm{~m})$ & $8 \times 10^{-4}$ & $3,7.5$ & 1 & \multicolumn{3}{|c|}{$10^{9}$} \\
\hline Diameter $(d)(\mathrm{mm})$ & 0.08 & 0.19 & 0.15 & \multicolumn{3}{|c|}{5000} \\
\hline f-number $\left(F_{\text {no }}\right)$ & 1 & 1600,4000 & 6700 & \multicolumn{3}{|c|}{$2 \times 10^{8}$} \\
\hline $\begin{array}{l}\text { Theoretical diffraction-limited } \\
\text { resolution: } \quad \text { Spatial }(\mu \mathrm{m}) \\
\\
\text { Angular (arc sec) }\end{array}$ & 0.035 & 0.3 & 1.2 & $0.3 \times 10^{-6}$ & $0.12 \times 10^{-6}$ & $0.07 \times 10^{-6}$ \\
\hline
\end{tabular}

References: (a) Spector et al. (1999) (b) Chen et al. (1999) (c) di Fabrizio \& Gentili (1999).

telescope and of flux concentration, there is no reason to make $p$ much smaller than the detector spatial resolution. Considerations of electron scattering and stopping distance suggest that the detector spatial resolution is likely to be limited to about $1 \mathrm{~mm}$ at $1 \mathrm{MeV}$, scaling approximately as $E^{0.8}$.

Pitches finer than the detector resolution may nevertheless sometimes be useful. They may allow larger lenses or shorter focal length to be used because the finest pitch dictates the maximium diffraction angle and hence relates $f$ and $d$ :

$f=0.403 \times 10^{6}\left(\frac{p}{1 \mathrm{~mm}}\right)\left(\frac{d}{1 \mathrm{~m}}\right)\left(\frac{E}{1 \mathrm{MeV}}\right) \mathrm{km}$.

\section{Astronomical applications}

The diffractive techniques proposed here can be adopted over a very wide range - at least from $\sim 10 \mathrm{keV}$ to $\sim 10 \mathrm{MeV}$. To investigate their potential for gamma-ray astronomy, a number of different cases will be considered - a low energy gamma-ray system optimised for $200 \mathrm{keV}$, a medium energy example $(500 \mathrm{keV})$ and a lens optimised for working in the $847 \mathrm{keV}{ }^{56} \mathrm{Co}$ line. Although where possible a lens should be optimised for a particular energy, it will be seen that a given device can in fact function over a relatively wide band by changing the detector position.

Figure 2 shows that, even without resorting to exotic materials such as Beryllium, losses in a gamma-ray PFL need only be a few percent. Selecting Aluminium, a low technology, low cost material, one finds $t_{\max }\left(=2 t_{\pi}\right)$ between 0.45 and $1.9 \mathrm{~mm}$ (Table 1 ) for the energies considered here. The transmission loss is only 1.5 to $2 \%$, even allowing for a $0.25 \mathrm{~mm}$ backing for constructional purposes (Fig. 3).

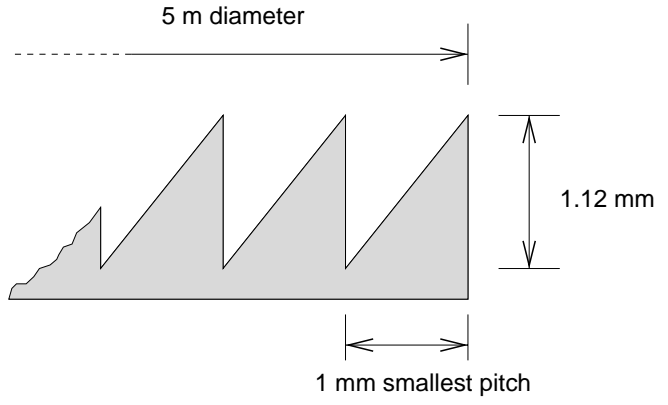

Fig. 3. Cross-section through the extreme edge of the aluminium disk forming the example $500 \mathrm{keV}$ gamma-ray PFL in Table 1.

As discussed above, the detector spatial resolution at these energies will be of the order of a millimetre, so from an angular resolution point of view there is no reason to consider very small values of $p$. This leads to the concept of a very simple lens in which a millimetre scale groove structure is machined into an aluminium plate a millimeter or so thick. The profile for the $500 \mathrm{keV}$ example lens with $p=1 \mathrm{~mm}$ is illustrated in Fig. 3 .

The main problem is that for any reasonable diameter the focal length becomes very long indeed. The $5 \mathrm{~m}$ diameter $500 \mathrm{keV}$ lens example (Tables 1, 2, Fig. 3) would have a focal length of just over $10^{6} \mathrm{~km}$ at $511 \mathrm{keV}$. But is this impracticable?

The Xeus studies (Bavdaz et al. 1999) have demonstrated the feasibility of having a focussing optic on one spacecraft and a focal plane assembly on another one actively controlled to remain at the focal point. The separation in the case of Xeus is only $50 \mathrm{~m}$ but this is in low earth orbit where gravity gradient effects are serious.

Many other mission concepts under study require precision control of the relative positions of spacecraft and the concept of "formation flying" has been validated by 
several studies. In fact there are engineering groups at JPL, GSFC and Estec that specialize in it. In particular the LISA gravitational wave observatory mission (LISA Study Team 1998) plans to use three spacecraft with distances of $5 \times 10^{6} \mathrm{~km}$ between each pair. The entire cluster will orbit the sun at $1 \mathrm{AU}$. The LISA spacecraft will be actively controlled to hold their position with respect to a proof mass within each to a precision of $\sim 1 \mathrm{~nm}$ (on a $1 \mathrm{~s}$ timescale).

This suggests that a stable baseline a few million $\mathrm{km}$ long between two spacecraft is not inconceivable and we will proceed to work through the implications of such a design.

\section{Practicalities, problems and anticipated performance}

\subsection{Mission considerations}

A major advantage of the proposed scheme is that in an optical system with a single lens, the image is to first order independent of any tilt of the lens with respect to the line joining the object-centre to the lens-centre and the image-centre. The accuracy with which the lens module must keep the axis of the lens pointed towards the target is only that needed to avoid aberrations due to using the lens too far off-axis. The formulae derived by Young (1972) for the aberrations of zone plates show that field curvature is the dominant effect in the case under consideration here and that it is insignificant for off-axis angles of less than a degree. Thus the pointing requirement for the lens module is trivial. Similarly the orientation of the detector module needs only to be controlled accurately enough that the distant lens is within the field of view of the collimator which would be needed to restrict the diffuse cosmic background. Thus for the detector, too, $\sim 1$ degree pointing precision should be sufficient.

The distance between the two spacecraft does not have to be controlled with great precision. An error of $1000 \mathrm{~km}$ in $10^{6} \mathrm{~km}$ will only lead to a $0.1 \%$ change in the energy for which the instrument is optimally focussed.

It is the relative position of the two spacecraft, transverse to the axis, which is critical. In terms of spacecraft position control, the requirements for the system under consideration here differ from those of LISA mainly in that the objective here is to maintain a stable position in true inertial space instead of within a local pseudo-potential in the solar system. The crucial issue is the control of the direction in space of the line joining the two spacecraft. The problem can be considered in terms of maintaining the detector array at the focal point of the lens despite gravity gradient forces, though in practice the lens carrying spacecraft could equally be the actively controlled one. For spacecraft separated by $f$ from each other and at $1 \mathrm{AU}$ from the sun and distance $d$ from the earth, gravity gradient forces correspond to accelerations in $\mathrm{m} \mathrm{s}^{-2}$ of

$4 \times 10^{-5} \alpha_{1}\left(\frac{f}{10^{6} \mathrm{~km}}\right)$ and

$4 \times 10^{-7} \alpha_{2}\left(\frac{f}{10^{6} \mathrm{~km}}\right)\left(\frac{d}{10^{7} \mathrm{~km}}\right)^{-3}$

due to the Sun and the Earth respectively. Here $\alpha_{1}, \alpha_{2}$ are factors in the range 1-2 which depend on the orientation. For systems on the scale considered here, one cannot simply place them at a Lagrangian point of the Earth-Sun system, but with $d<10^{7} \mathrm{~km}$ and with careful mission planning the gravitational forces due to the earth may partly counteract the solar gravity gradient and could help in telescope repointing.

Solar radiation pressure produces an acceleration $4.5 \times$ $10^{-6} \alpha_{3}(A / M) \mathrm{m} \mathrm{s}^{-2}$, on a spacecraft of mass $M(\mathrm{~kg})$ presenting area $A\left(\mathrm{~m}^{2}\right)$ to the solar flux. Here $\alpha_{3}$ is another factor in the range 1-2. For long focal length systems and for conventional spacecraft proportions, the effects of radiation pressure will usually be less important than those due to gravity gradient effects, particularly as it is only the differential force which matters. It is noted however that solar sails with an area of a few thousand square metres might be used to help minimise the active propulsion requirements.

The accelerations are such that for a spacecraft of a few hundred $\mathrm{kg}$, thrusts of about $10 \mathrm{mN}$ are required. This is an order of magnitude less than needed for Xeus and is well matched to the capability of ion thrusters developed for geosynchronous satellite station keeping. The integrated momentum changes necessary to counteract these forces and to allow for multiple telescope pointings are of the order of $1 \mathrm{~km} \mathrm{~s}^{-1} \mathrm{y}^{-1}$ and $10 \mathrm{~km} \mathrm{~s}^{-1} \mathrm{y}^{-1}$; these, too, are much less than the requirement for Xeus and are comparable with those needed to deploy the LISA array. Thus these requirements do not seem entirely unreasonable.

\subsection{Attitude determination and control}

The relative positions of the spacecraft in a direction transverse to the line of sight need to be known to a precision of a few millimetres and controlled better than, say, $10 \mathrm{~cm}$. As discussed above, the dominant disturbing forces are gravitational. Because the potential within the inner solar system is extremely well understood they can be accurately predicted. Clearly a closed loop control system will be needed however to avoid errors accumulating. The disturbances will change only slowly and the timescale over which the feedback loop will have to operate will depend mainly on uncertainties in thrusters. By using data from accelerometers, it should be possible to reduce these uncertainties to a low enough level that the main feedback loop can be a slow one.

Providing an absolute reference is the biggest challenge presented by the proposed approach, though it is no worse than for any other mission aiming at micro arcsecond resolution.

The HST fine guidance system uses interferometric sensors which have milli arcsecond performance but which 
Table 2. Expected performance and comparison with other missions.

\begin{tabular}{|c|c|c|c|c|c|}
\hline & $\begin{array}{c}\text { Integral SPI } \\
\text { Spectrom. }\end{array}$ & $\begin{array}{l}\text { Integral Ibis } \\
\text { Imager }\end{array}$ & \multicolumn{3}{|c|}{$\begin{array}{c}\text { Example Diffractive Lens } \\
\text { Telescopes }\end{array}$} \\
\hline Focal length $(\mathrm{m})$ & 1.7 & 3.2 & \multicolumn{3}{|c|}{$10^{9}$} \\
\hline $\begin{array}{l}\text { Band }(\mathrm{keV}) \text { - fixed configuration } \\
\text { Band }(\mathrm{keV}) \text { for } 50 \% \text { response } \\
\text { with separation adjusted }\end{array}$ & $20-8000$ & $15-10000$ & $\begin{array}{c}200 \pm 0.8 \\
125-500\end{array}$ & $\begin{array}{l}500 \pm 0.9 \\
325-1200\end{array}$ & $\begin{array}{l}847 \pm 1.0 \\
540-2100\end{array}$ \\
\hline Effective area $\left(\mathrm{m}^{2}\right)^{(1)}$ & 0.009 & 0.05 & 12.1 & 6.4 & 4.4 \\
\hline $\begin{array}{l}\text { Angular resolution }\left(\mu^{\prime \prime}\right) \\
\quad \text { (intrinsic) } \\
\text { (with chromatic aberration) }\end{array}$ & $10^{10}$ & $10^{9}$ & $\begin{array}{l}0.3 \\
1.7\end{array}$ & $\begin{array}{c}0.12 \\
0.7\end{array}$ & $\begin{array}{c}0.07 \\
0.5\end{array}$ \\
\hline $\begin{array}{l}\text { Sensitivity }^{(2)} \\
\\
\text { Continuum }^{(3)} \\
\text { Narrow Line }\end{array}$ & $2.5 \times 10^{-5}$ & $1.6 \times 10^{-6}$ & $\begin{array}{l}2.5 \times 10^{-9} \\
8 \times 10^{-10}\end{array}$ & $\begin{array}{c}4 \times 10^{-9} \\
1.5 \times 10^{-9}\end{array}$ & $\begin{array}{l}4.5 \times 10^{-9} \\
1.8 \times 10^{-9}\end{array}$ \\
\hline
\end{tabular}

(1) At $500 \mathrm{keV}$ for SPI/Ibis; Detector efficiency and $20 \%$ provision for lens imperfections taken into account.

(2) Sensitivities are for a point source. For the example PFL telescopes, the background taken is based on SPI predictions, scaled to $1 \mathrm{~cm}^{2}$, corresponding to $2 \mu^{\prime \prime}$.

(3) Photons $\mathrm{cm}^{-2} \mathrm{~s}^{-1} \mathrm{keV}^{-1}$ for $5 \sigma$ in 1 day, $E=500 \mathrm{keV}, \mathrm{d} E=250 \mathrm{keV}$ (Ibis).

(4) Photons $\mathrm{cm}^{-2} \mathrm{~s}^{-1}$ for $5 \sigma$ in $10^{6} \mathrm{~s}$, (SPI figure is for $500 \mathrm{keV}$ ).

rely on the primary optical system and even were it precise enough this does not offer an economic approach to the problem. The same applies a fortiori to the system planned for NGST. The MAXIM study plans to rely on developments in the optical band underway for the Space Interferometry Mission, SIM, and this would be one approach.

Alternatively, given the centimetre scale precision needed in the case under consideration here, it is natural to turn to the radio band and it would appear that a solution can probably be found using radio navigation signals (J. Bar-Sever, private communication). For example one could consider using phase-locked radio beacons on the two spacecraft, which are in contact with each other and with at least 3 ground stations. The ground stations would probably have to use radio astronomical observations to establish their positions with respect to a reference frame defined by distant objects.

The positions of the targets will have to be known sufficiently well that they can be found. The main objective of SIM is to perform micro arcsecond astrometry (using resolution much lower than proposed here, but taking advantage of centroiding) and this could provide an adequate frame of reference.

It is noted that it could turn out to be best to use radio astronomical measurements direct from the two spacecraft to establish the pointing with respect to a celestial reference frame and that a radio interferometer using receivers at each end of this long stable baseline would itself be a powerful astronomical tool.

\subsection{Chromatic aberration}

Chromatic aberration is an important limiting factor for PFLs. With the detector plane in the focal position for the nominal design energy, the focal spot becomes more and more blurred as one considers energies further and further from the nominal.

Simulation shows that there is a contribution to the $60 \%$ power diameter corresponding to an angular resolution (in radians) of about

$0.2(\Delta E / E)(d / f)$.

This will exceed the diffraction limited component if $(E / \Delta E)<d^{2} /(6 f \lambda)$ (the exact numerical constant will depend on the definitions of resolution and of bandwidth used). As the number of cycles (rings) in the lens pattern is given by $N=d^{2} / 8 f \lambda$, this implies that chromatic aberration will dominate if $(E / \Delta E)<\sim N$. This will often be the case - the $500 \mathrm{keV}$ example case considered here has 1250 cycles but even a very good Germanium detector will have $(E / \Delta E)<300$ at $500 \mathrm{keV}$.

In Paper II techniques for increasing the bandwidth will be discussed, but for the present we accept that (for continuum radiation) chromatic aberration may be a limiting factor when trying to achieve the very highest angular reolution.

Some of the advantage of the large collecting area is lost by having to work within a narrow bandwidth. However, provided the observations are detector background noise limited, from the point of view of sensitivity as well as that of angular resolution, $\Delta E$ should be as narrow as the detector resolution will allow. This is true even for continuum studies. It arises because if the bandwidth is large, so that the above limit on $(E / \Delta E)$ is not met, the angular resolution will be degraded. For a point source data will then have to be collected from a larger region of the detector plane and this will normally result in increased background noise. Assuming that the background in an area $A_{\text {pix }}$ is proportional to $A_{\text {pix }} \Delta E$, the associated Poissonian noise will increase in proportion to $\Delta E^{3 / 2}$. 

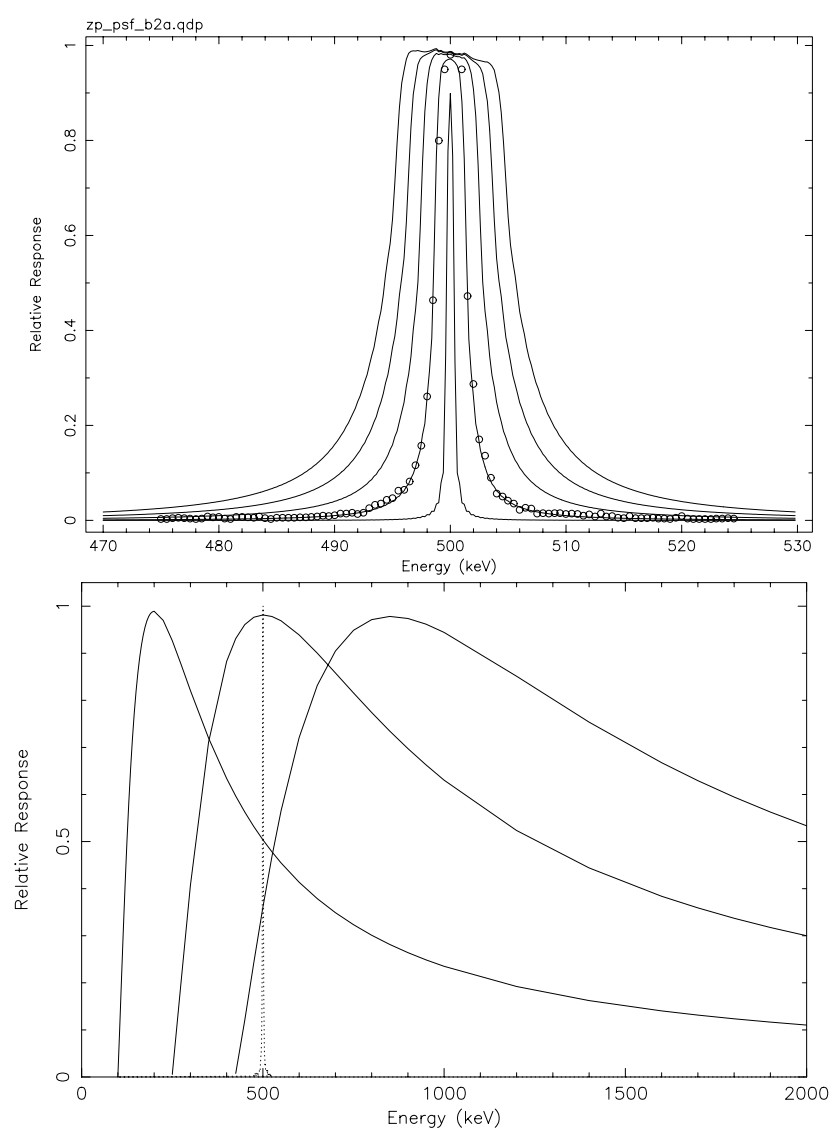

Fig. 4. a) The predicted response as a function of energy of a PFL lens optimised for one energy of $500 \mathrm{keV}$ but used at different energies. The lens parameters are as in Table 2 . The signal in a detector pixel of 2 (narrowest), 10, 20, 30, $40 \mathrm{~mm}$ diameter is shown. The curves are computed using a method based on the work of Lommel (Boivin 1952) and do not take into account absorption. Results from a Monte Carlo simulation with absorption taken into account are shown for the $10 \mathrm{~mm}$ case and show that the corrections are small. b) The response of PFLs optimised for 200, 500 and $847 \mathrm{keV}$ if the detector is moved to an appropriate focal position for each energy. (Monte Carlo method; absorption effects included) The curve from a) for a $10 \mathrm{~mm}$ detector is shown dashed for comparison.

As signal from a continuum source will only increase as $\Delta E$, small values of $\Delta E$ are preferable.

This is one respect in which the approach discussed here is at a disadvantage compared with the interferometric system proposed for MAXIM, which operates over a wide band (data from different energies can be combined because the detector is energy resolving and the point source response function at each energy is known).

Although a PFL will be optimised for a particular energy, that does not preclude its use at other energies by moving the detector to the corresponding focal plane at $f^{\prime}=f E^{\prime} / E$. Figure $4 \mathrm{~b}$ shows how the efficiency varies if a lens is refocussed for a different energy in this way. Over a band of energy spanning a factor of 2-3, the effective area is reduced by less than a factor of two.

\subsection{Optical tolerances}

The fact that the refractive index is extremely close to that of vacuum means that the lens performance is relatively immune to problems of surface roughness, material inhomogeneities and constructional tolerances. Attaining $\lambda / 10$ phase precision requires no better than $0.1 \mathrm{~mm}$ quality for a $500 \mathrm{keV}$ aluminium lens, for example. Temperatures can change by some tens of degrees without serious effect.

\subsection{Anticipated performance}

Depending as it does on simple physics and tolerance requirements which are far more lax than the state of the art, the performance of the lens can be predicted with confidence. The pessimistic assumption of $80 \%$ efficiency still leaves about $16 \mathrm{~m}^{2}$ effective area for the example lens. In making estimates of sensitivity in the remainder of this work, the photopeak efficiency and background expected for the SPI Germanium Spectrometer on Integral are used. For example, at $500 \mathrm{keV}$ these are $42 \%$ and $2.5 \times 10^{-5}$ events $\mathrm{cm}^{-3} \mathrm{~s}^{-1} \mathrm{keV}^{-1}$. With a germanium detector working at this energy those events within an energy window of $\Delta E / E=0.5 \%$ can be selected. The corresponding chromatic aberration will increase the focal spot size from $0.6 \mathrm{~mm}$ to $5 \mathrm{~mm}$, limiting the angular resolution to about $1.4 \mu^{\prime \prime}$. The resulting sensitivity and the corresponding values for the other example systems are given in Table 2 .

\section{Discussion and examples of capabilities}

We now consider the capabilities of telescopes using the proposed techniques to tackle some of the astrophysical objectives which gave rise to the studies described here. In assessing the feasibility of directly imaging the emission from an object, two considerations arise - if the angular scale is too small it will not be resolved and only a total flux measurement will be obtained. If it is too large then the surface brightness will be insufficient for a useful detection.

Ignoring the small losses in the lens, the surface brightness of the image of a resolved object will be $\sim(d / f)^{2}$ of that of the object. This ratio is $2 \times 10^{-17}$ for the example systems considered here. For an observation of at least a few days, significant detection will be obtained if the surface density of the detected photons at the detector is comparable with with the background event density (because of the small detector and narrow bandwidth the telescope would be signal limited and not background limited for shorter observations). Again using the expected Integral SPI background, this requirement corresponds to an object with a brightness temperature at $500 \mathrm{keV}$ exceeding $k T=8.1 \mathrm{keV}$ (or $3.2 \mathrm{keV}, 13.7 \mathrm{keV}$ at 200 , $847 \mathrm{keV}$ ). Athough one is far from the Rayleigh-Jeans regime, this is in some circumstances still a useful yardstick. A black body with these temperatures and large enough to be resolved would be have a luminosity orders 
of magnitude greater than any conceivable astrophysical object, whatever distance scale is considered. Thus observable resolved sources will be in practice be optically thin and/or non-thermal.

\subsection{Imaging Black Hole systems in AGNs}

The origin of the gamma-ray emission from Active Galactic Nucleii (AGNs) is poorly understood. Indeed the possibility of direct visualisation of this process to understand what is going on was one of the incentives for the studies presented here.

For Seyferts, it is generally accepted that the emission at a few hundred $\mathrm{keV}$ arises from Comptonisation of soft photons, but whether the Comptonising electrons are thermal or non-thermal, and to some extent the size of the region in which the Comptonisation occurs, is less certain. Several characteristic scales can be recognised:

- The event horizon $R_{\mathrm{H}}=0.5-1 R_{\mathrm{S}}$, depending on the spin, where $R_{\mathrm{S}}$ is the Schwarzschild radius of the black hole.

- The inner radius of the accretion disk, which can be supposed to be equal to the radius of the innermost stable orbit - between $3 R_{\mathrm{S}}$ for a non-rotating (Schwarzschild) Black Hole and $0.62 R_{\mathrm{S}}$ for a maximally rotating Kerr Black Hole.

- The size of the shadow of the black hole defined by the apparent size of the boundary between the regions of a source behind the black hole which can reach the observer and those that cannot. For a Schwarzschild Black Hole this is $5.2 R_{\mathrm{S}}$ (Falcke et al. 2000).

- The outer radius of the accretion disk, which is probably an order of magnitude greater than its inner radius.

Where jets are involved, the gamma-ray emitting region could be on the same size scale as the accretion disk or could be much larger.

Thus the resolution needed to study such systems is likely to be from one to a fews tens of Schwarzschild radii.

There have now been estimates made of the central mass in numerous galaxies, by reverbration mapping and other techniques. Figure 5 shows how these estimates are distributed as a function of redshift $z$ or, in the case of nearby objects, of distance expressed as an equivalent redshift $z=d H_{0} / c$. The plot also shows the locus of points for which 1 micro arcsecond angular resolution would correspond to one Schwarzschild radius and to 100 Schwarzschild radii. Clearly the objects for which estimates of the central mass are available are in no way an unbiased sample, but Fig. 5 gives an indication of the region of parameter space which is most likely to be most interesting.

Few of the objects for which central mass estimates are available have been detected in the gamma-ray band. This probably results from a combination of selection effects and the limited sensitivity of current gamma-ray instruments. Present measurements provide little indication of how many AGN might be detectable with a factor of
1000 improvement in sensitivity. To help in addressing this question, Fig. 5 shows (as dashed lines) the locii of objects which would be just detectable if emitting gamma-rays at particular fractions $f$ of their Eddington limits.

\subsection{The special case of Sgr A*}

The mass of the black hole in Sgr $A^{*}$ is now thought to be $(2.6-3.3) \times 10^{6} M_{\odot}$ and the angular equivalent of $R_{\mathrm{S}}$ is thus $6 \mu^{\prime \prime}$. There has been continued interest in trying to take advantage of the poximity of $\operatorname{Sgr} A^{*}$ to enable sensitive probing of massive black hole systems. Falcke et al. (2000), for example, have discussed the feasibility of viewing the shadow of the black hole using sub-millimeter VLBI observations, with $16 \mu^{\prime \prime}$ resolution, which should be possible if VLBI measurements can be made at $\lambda=$ $0.6 \mathrm{~mm}$. At first site Sgr $\mathrm{A}^{*}$ appears to be an obvious target for a PFL gamma-ray telescope.

Gamma-ray emission from Sgr A* is known to be weak, except possibly in the Egret $(\mathrm{GeV})$ band. The best models for the emission from $\mathrm{Sgr} \mathrm{A}^{*}$ over wide range of energy are probably those based on Advection Dominated Accretion Flows (ADAFs) (Lasota 1999). The currently available ADAF models (Narayan et al. 1998) are based on estimates of the X-ray flux from Rosat. Of the range of parameters considered by Narayan et al. recently available results from Chandra (Baganoff et al. 2001) suggest that the lowest luminosity combinations should be taken. However the sensitivity at $200 \mathrm{keV}$ shown in Table 2 is such that even on this assumption a $\sim 100 \sigma$ detection should be obtained in 1 day.

The most serious problem is that the source is likely to be be over-resolved. If emission is from a disk of $10 R_{\mathrm{S}}$, the signal will be spread over 2000-3000 pixels and even sophisticated modelling techniques would barely offer a simple detection. In these circumstances there is some advantage in collecting data from a wider bandwidth and accepting the resulting degradation in resolution, but the factor gained is not large. It is probably better to consider lower energies and a shorter focal length for objects so weak and so "large". Variations on the PFL technique which may help with this problem will be discussed in Paper II but MAXIM will be well suited to such observations.

Even at X-ray wavelengths there may be surface brightness problems if there is outflow. Ozel \& Di Matteo (2001) have shown that in low luminosity models, particularly those with high outflow parameters, the low energy $\mathrm{X}$-ray emission can extend to large radii, even to $10^{6} R_{\mathrm{S}}$ at $1 \mathrm{keV}$.

\subsection{Studies of gamma-ray emission from around hot stars}

Inverse Compton gamma-rays are expected to be produced as the particles accelerated in the instabilities in winds around $\mathrm{OB}$ supergiants interact with the stellar uv photons (Chen \& White 1991a,b). Such a star at $5 \mathrm{kpc}$ would have an apparent radius of more than $10 \mu^{\prime \prime}$ and 


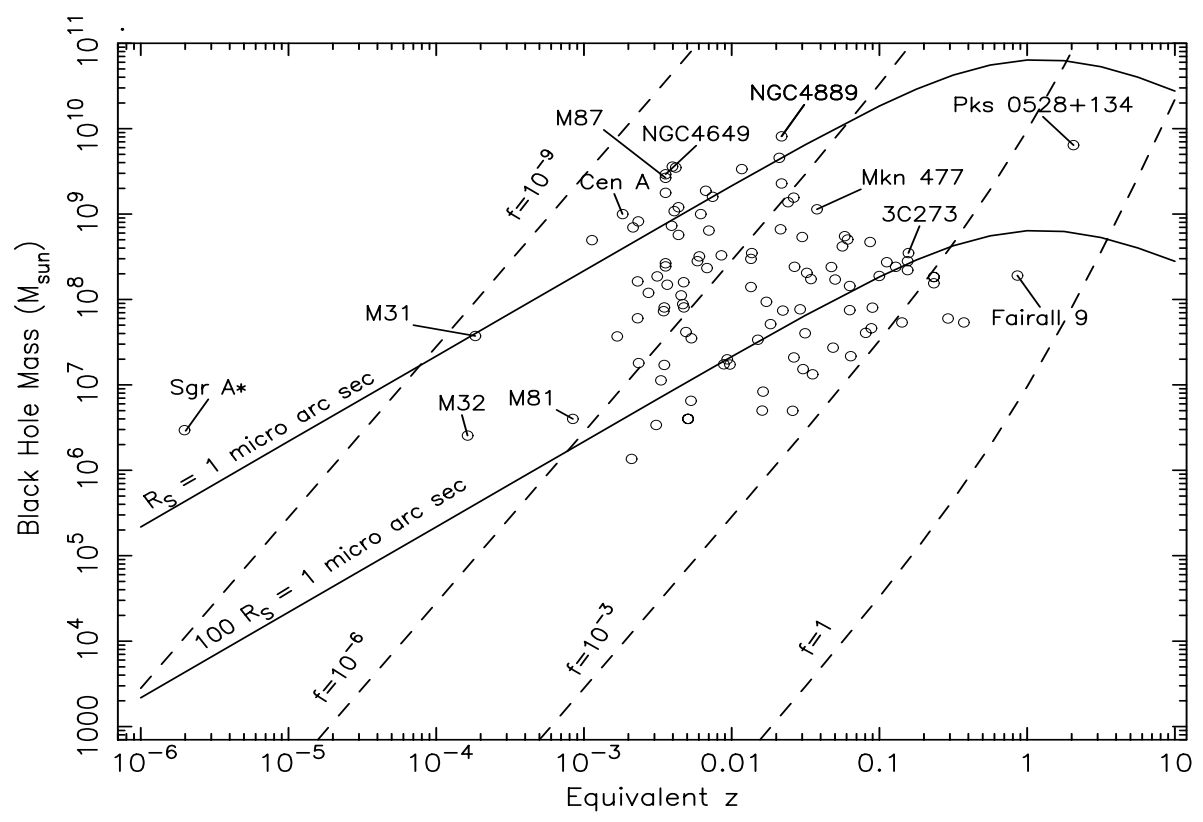

Fig. 5. Estimates from the literature of masses of the central object in AGN and other galaxies. The estimates are plotted as a function of a distance measure (see text). Solid lines show the locii of points for which the Schwarzschild radius has a particular angular scale and dashed lines show the locii of objects which would be detectable if emitting at a specific fraction of their Eddington limit. The $5 \sigma$ in 1 day point source sensitivity of the $200 \mathrm{keV}$ telescope in Table 1 is taken and $\nu F_{\nu}$ at $200 \mathrm{keV}$ in the

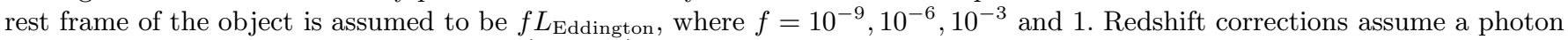
index of $-2 . H_{0}$ is taken to be $70 \mathrm{~km} \mathrm{~s}^{-1} \mathrm{Mpc}^{-1}$ and $q_{0}=1 / 2$. Mass estimates are from various sources and compilations (Ferrarese \& Merritt 2000; Franceschini et al. 1998; Fromerth \& Melia 2000; Gebhardt \& Bender 2000; Gebhardt et al. 2000; Kaspi et al. 2000; Magorrian et al. 1998; Marconi et al. 2000; Merritt \& Ferrarese 2001; Nelson 2000; Nishiura \& Taniguchi 1998; Peterson \& Wandel 2000; Wandel 1999); where multiple estimates exist the geometrical mean is plotted.

the emission around it could be directly imaged with a PFL telescope, allowing detailed study of the instabilities and the acceleration processes.

When massive hot stars are in close binary systems, the colliding winds are likely to produce strong gamma-ray sources (Eichler \& Usov 1993; Pittard \& Stevens 1997). For binary periods of a few days, and source distances of a few $\mathrm{kpc}$, the angular scale of the system will be of the order of $10 \mu^{\prime \prime}$, well matched to the capabilities of a PFL telescope.

There is also the possibility of directly imaging the particle acceleration in the instabilities in the winds in, for example, OB supergiants.

\subsection{Stellar coronal activity and flares}

With micro arcsecond resolution, stellar disks will be resolvable even at large distances (a $1 M_{\odot}$ main sequence star will submit $1 \mu^{\prime \prime}$ at $4.6 \mathrm{kpc}$ ). High energy activity in stellar coronae could be directly imaged.

\subsection{Line emission from supernovae}

The extremely good sensitivity resulting from the large collecting area and flux concentrating capabilities of systemns using the principles proposed here mean that they are of interest even where the target may be unresolved. An example of this is in studying gamma-ray emission from supernovae. The analysis of
(Timms \& Woosley 1997) shows that a broad line sensitivity of $2 \times 10^{-6} \gamma \mathrm{s}^{-1} \mathrm{~cm}^{-2}$ would be needed to detect the $847 \mathrm{keV}^{56} \mathrm{Co}$ line from at least one Type Ia supernova per year. This is at the limit of the capabilities of the projected ATHENA mission and an order of magnitude more sensitive than INTEGRAL. An instrument with parameters of the $847 \mathrm{keV}$ example in Table 2 would have a broad line sensitivity of $1.5 \times 10^{-8} \gamma \mathrm{s}^{-1} \mathrm{~cm}^{-2}$ ( $5 \sigma$ in $10^{6} \mathrm{~s}$ ) so it would be able to detect gamma-ray emission out to $z=0.1$.

The line emission from Type II will be much narrower but much weaker; SN1987a was only detected in gammarays because of its extreme proximity. Nevertheless, the narrow line sensitivity of the instruments discussed here $\left(2 \times 10^{-9} \gamma \mathrm{s}^{-1} \mathrm{~cm}^{-2}\right)$ would, on the basis of the calculations of Timms and Woosely, allow the detection of most Type II supernovae within $70 \mathrm{Mpc}$.

Even the use of the imaging properties of the telescope to study the spread of the ejecta from a Type Ia supernova is conceivable for events within about $50 \mathrm{Mpc}$ ( $\sim 1$ per year), which attain diameters of several micro arcseconds in a few months and for which the $>100 \sigma$ signal would allow the imaging properties to be used to good advantage.

\section{Variations on the basic approach}

An obvious question is whether a similar approach could be adopted at X-ray energies. For a lens of similar 
diameter, the diffraction limited angular resolution will be $\sim 100$ times worse, but for some applications this might be appropriate. These issues and ways in which the focal length may be reduced or the bandwidth widened will be discussed in Paper II.

\section{Conclusions}

Moving up in energy to the gamma-ray band apparently simplifies a number of problems in the search for sub micro arcsecond resolution. The proposed system is very long (millions of $\mathrm{km}$ ) but the fabrication of the optics would be almost trivial and there is no need for critical alignment of the individual components. Only the pointing of the telescope as a whole is critical and it is suggested that the requirements of the pointing determination system are well matched to techniques using radio measurements, while the associated active control seems to be possible with existing technology.

Compared with proposals for achieving microarcsecond resolution at X-ray energies using interferometry, the approach suggested here has the disadvantage that it only operates over a narrow energy band at any one time. On the other hand, the optics are simple and are capable of concentrating the flux from a very large aperture onto a detector area $10^{4}-10^{7}$ times smaller in area and so the sensitivity can be extremely good despite the small bandwidth. Furthermore, in the gamma-ray band micro arcsecond resolution can be obtained with modest diameter. For those objects which are gamma-ray sources as well as X-ray sources, the approach seems to be very promising.

Acknowledgements. The author is grateful for helpful comments and suggestions from J. Bar-Sever (JPL), J. Brown (University of Glasgow), N. Gehrels (GSFC) and colleagues at both the University of Birmingham and CESR, Toulouse.

\section{References}

Baganoff, F., Maeda, Y., Morris, M., et al. 2001, ApJ, to be published

Bavdaz, M., Beijersbergen, M. W., Peacock, A. J., et al. 1999, Proc. of SPIE, 3766, 94

Boivin, A. 1952, J. Opt. Soc. Am., 42, 60

Cash, M., Shipley, A., Osterman, S., \& Joy, M. 2000, Nature, 407, 160

Chantler, C. T. 1995, J. Phys. Chem. Ref. Data, 24, 71

Chen, W., \& White, R. L. 1991a, ApJ, 381, L63

Chen, W., \& White, R. L. 1991b, ApJ, 366, 512

Chen, Z., Vladimirsky, Y., Brown, M., et al. 1999, J. Vac. Sci. Tech., B 17, 2522

Craig, W. W., Christensen, F. E., Decker, T. A., et al. 1998, Proc. of SPIE, 3445, 112

Desai, U. D., Orwig, L. E., Piquet, L., \& Gaither, C. C. 1998, Proc. of SPIE, 3442, 94

di Fabrizio, E., \& Gentili, M. 1999, J. Vac. Sci. Tech., B 17, 3439

Eichler, D., \& Usov, V. 1993, ApJ, 402, 271
Falcke, H., Melia, F., \& Agol, E. 2000, ApJ, 528, L13

Ferrarese, L., \& Merritt, D. 2000, ApJ, 539, L9

Franceschini, A., Vercellone, S., \& Fabian, A. C. 1998, MNRAS, 297, 817

Fromerth, M. J., \& Melia, F. 2000, ApJ, 533, 172

Fujisaki, H., \& Nakagiri, N. 1990, Appl. Opt., 29, 483

Gebhardt, K., Bender, R., Bower, G., et al. 2000, ApJ, 539, L13

Gebhardt, K., Kormendy, J., Ho, L. C., et al. 2000, ApJ, 543, L5

Gorenstein, P., Joensen, K. D., Romaine, S. E., et al. 1996, Proc. of SPIE, 2807, 119, see

http://heasarc.gsfc.nasa.gov/docs/heasarc/concepts /hxrt.html

Hussain, A. M., Christensen, F. E., Jimenez-Garate, M. A., et al. 1999, Proc. of SPIE, 3766, 184

Jerius, D., Donnelly, R. H., Tibbetts, M. S., et al. 2000, Proc. of SPIE, 4012, 17

Kaspi, S., Smith, P. S., Netzer, H., et al. 2000, ApJ, 533, 631

Kraemer, G., Einighammer, H. J., Elwert, G., et al. 1978, Sol. Phys., 57, 345

Laporte, P., Kohnle, A., Abrosimov, N. et al. 1999, Astrophys. Lett. Commun., 39, 453

Lasota, J. 1999, Phys. Rep., 311, 247

LISA Study Team 1998, LISA Pre-Phase A Report, Vol. 2nd edition, revised (Garching: Max-Planck-Institut fur Quantenoptik)

Magorrian, J., Tremaine, S., Richstone, D., et al. 1998, AJ, 115,2285

Mao, P. H., Harrison, F. A., Windt, D. L., \& Christensen, F. E. 1999, Appl. Opt., 38, 4766

Marconi, A., Schreier, E. J., Koekemoer, A., et al. 2000, ApJ, 528,276

Merritt, D., \& Ferrarese, L. 2001, MNRAS, 320, L30

Mertz, L., \& Young, N. 1961, in Proc. Int. Conf. on Optical Instrum., London, 1961, ed. K. J. Habell (Chapman \& Hall), 305-312

Miyamoto, K. 1959, J. Opt. Soc. Am., 51, 17

Narayan, R., Mahadevan, R., Grindlay, J., Popham, R., \& Gammie, C. 1998, ApJ, 492, 554

Nelson, C. H. 2000, ApJ, 544, L91

Nishiura, S., \& Taniguchi, Y. 1998, ApJ, 499, 134

Ogasaka, Y., Yamashita, K., Serlemitsos, P. J., et al. 2000 , Adv. Space Res., 25, 911

Ozel, F., \& Di Matteo, T. 2001, preprint

Peterson, B. M., \& Wandel, A. 2000, ApJ, 540, L13

Pittard, J. M., \& Stevens, I. R. 1997, MNRAS, 292, 298

Skinner, G. K. 2001a, in preparation

Skinner, G. K. 2001b, PASP, Proc. IAU, 205

Soret, J. L. 1875, Annalen der Physik und Chemie, 156, 99

Spector, S. J., Jacobsen, C. J., \& Tennant, D. M. 1999, J. Vac. Sci. Tech., B 15, 2872

Stockman, Y., Hansen, H., Houbrechts, Y., et al. 1999, Proc. of SPIE, 3739, 298

Timms, F., \& Woosley, S. 1997, ApJ, 489, 160

Wandel, A. 1999, ApJ, 519, L39

White, N. E., \& Tananbaum, H. 1999, Astron. Nachr., 320, 280

Wood, R. W. 1898, Phil. Mag., 45, 511

Yamashita, K., Serlemitsos, P. J., Tueller, J., et al. 1998, Appl. Opt., 37, 8067

Yang, B. X. 1993, Nucl. Instrum. Meth. Phys. R., A 328, 578

Young, M. 1972, J. Opt. Soc. Am., 62, 972 